\title{
A case of systemic melioidosis: unravelling the etiology of chronic unexplained fever with multiple presentations
}

The authors declare no financial disclosure

\begin{abstract}
Melioidosis, caused by the environmental saprophyte, Burkholderia pseudomallei, is an important public health problem in Southeast Asia and Northern Australia. It is being increasingly reported from other parts, including India, China, and North and South America expanding the endemic zone of the disease. We report a case of systemic melioidosis in a 58-year-old diabetic, occupationally-unexposed male patient, who presented with chronic fever, sepsis, pneumonia, pleural effusion and subcutaneous abscess, was undiagnosed for long, misidentified as Pseudomonas aeruginosa infection elsewhere, but was saved due to correct identification of the etiologic agent and timely institution of appropriate therapy at our institute. A strong clinical and microbiological suspicion for melioidosis should be considered in the differential diagnosis of acute pyrexia of unknown origin, acute respiratory distress syndrome and acute onset of sepsis, especially in the tropics.
\end{abstract}

Key words: melioidosis, Burkholderia pseudomallei, diabetes mellitus, systemic infection, pleural effusion

Pneumonol Alergol Pol 2016; 84: 121-125

\section{Introduction}

Melioidosis or "the remarkable imitator", an acute infectious disease, caused by the environmental Gram-negative bacillus, Burkholderia pseudomallei, is endemic, widespread and an important public health problem in Southeast Asia and Northern Australia [1, 2]. It is being increasingly reported from other parts of the world, including India, China, Sri Lanka, and North and South America expanding the endemic zone of the disease [3, 4].

We report a case of melioidosis in a diabetic, occupationally unexposed male, who presented with chronic fever, sepsis, pneumonia, pleural effusion and subcutaneous abscess, was undiagnosed for long, misidentified as Pseudomonas aeruginosa infection elsewhere, but was saved due to correct identification of the etiologic agent and timely institution of appropriate therapy at our institute.

\section{Case}

A-58-year-old male, office employee, was admitted to our hospital in mid-September 2015 with a history of unremittent high-grade fever with intermittent cough and expectoration for 3 months, a progressively increasing swelling on posterior left chest wall for two months, right-sided pleuritic chest pain for one month, and shortness of breath for 2 weeks. He had decreased appetite and progressive loss of weight for the past one month. He was a known diabetic since 20 years managed with oral hypoglycaemic agents. There was no history of travel outside his place of residence in the preceding 6 months of his onset of symptoms. However, when probed,

Address for correspondence: Dr. Baijayantimala Mishra, Department of Microbiology, All India Institute of Medical Sciences, Bhubaneswar, Odisha, India, Pin-751019, e-mail:bm_mishra@hotmail.com

DOI: 10.5603/PiAP.2016.0012

Received: 18.12 .2016

Copyright (C) 2016 PTChP

ISSN 0867-7077 


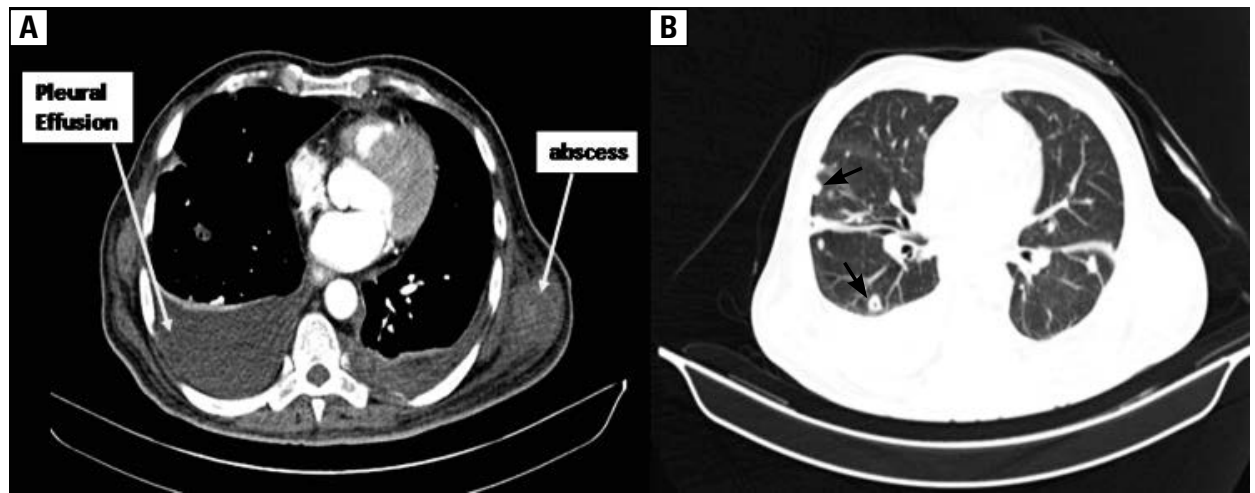

Figure 1. Contrast-enhanced chest CT showing (A) bilateral pleural effusions (right more than left) and left chest wall abscess without any pleuro-abscess communication, and (B) mediastinal lymphadenopathy with bilateral peripheral lung nodular opacities with cavitation

the patient provided a history of renovation of his toilet 15 days prior to the onset of symptoms, for which he was going to open fields for defecation, though with proper footwear.

Prior to his admission at our hospital, he had consulted four different hospitals and received several broad-spectrum antibiotics including carbapenems and antipseudomonal antibiotics based on the culture isolate of Pseudomonas auerogino$s a$ in the diagnostic pus aspirate from the chest wall swelling performed in the hospital he visited last. Empirical antitubercular therapy was also started in one of the hospitals, but was discontinued after a few days owing to gastrointestinal toxicity. Thus, despite a reasonable duration of antimicrobial therapy, he remained febrile with high temperature spikes $\left(>39^{\circ} \mathrm{C}\right)$ and his general condition progressively worsened.

Physical examination revealed a thin-built anemic man, conscious, but too sick and debilitated to take interest in the surrounding. He had weight loss of $7 \mathrm{~kg}$ since the start of his symptoms. Admission vital parameters were deranged with heart rate 120/min, blood pressure 108/66 mm Hg, respiratory rate $26 / \mathrm{min}$, oral temperature $40.0^{\circ} \mathrm{C}$ and oxygen saturation of $96 \%$ at room air. Patient had a pyogenic extra-thoracic abscess of $7 \times 4$ $\mathrm{cm}$ at left infra-scapular area. Chest auscultation revealed diminished breath sounds in right basal area with inspiratory crackles. Other systemic findings were normal.

\section{Laboratory investigations}

Total leucocyte count was $16.91 \times 10^{9} / 1(93 \%$ polymorphs, $6 \%$ lymphocytes, $1 \%$ monocytes), total red blood cell count $2.9 \times 10^{12} / 1$, platelet count $185 \times 10^{9} / \mathrm{l}$ and haemoglobin $65 \mathrm{~g} / \mathrm{l}$. Blood metabolic panel suggested poor diabetes control (random blood sugar $381 \mathrm{mg} / \mathrm{dl}$, fasting blood glucose $260 \mathrm{mg} / \mathrm{dl}$, postprandial blood sugar 274 $\mathrm{mg} / \mathrm{dL}$ ), with urea $26 \mathrm{mg} / \mathrm{dl}$ and creatinine $1 \mathrm{mg} / \mathrm{dl}$. Serum electrolytes and liver function tests were within normal limits except for a low serum albumin level of $1.4 \mathrm{~g} / \mathrm{dl}$.

Contrast enhanced computed tomogram (CT) of the thorax showed bilateral pleural effusions (right more than left), and left chest wall abscess without any pleuro-abscess communication (Fig. 1A). Soft-tissue window showed mediastinal lymphadenopathy and bilateral peripheral lung nodules with cavitations consistent with septic pulmonary emboli (Fig. 1B). A diagnosis of septicaemic bronchopneumonia with bilateral pleural effusions was made. Aseptically drained pus along with blood, urine, sputum, and pleural fluid ( $1.5 \mathrm{ml}$, right side aspirate) were subjected to microbiological investigations, including culture. The patient was commenced on empiric therapy with parenteral antibiotics (ceftazidime, 2 gm IV 8-hourly, and levofloxacin $750 \mathrm{mg}$ IV once-daily) as these antibiotics were not used earlier.

Blood peripheral smear demonstrated microcytic hypochromic anaemia without any parasites or abnormal cells. Sputum smear examination for acid-fast bacilli was negative. Gram stain of the pus aspirate showed polymorphs (6-8 per oil immersion field) and few gram-negative bacilli. Microscopy and biochemistry of the pleural fluid aspirate were supportive of an exudate (total leucocyte count -1250 cells per cubic millimetre; $50 \%$ polymorphs; protein $2.5 \mathrm{~g} / \mathrm{dl}$; glucose 130 $\mathrm{mg} / \mathrm{dl}$; Lactate dehydrogense $230 \mathrm{IU} / \mathrm{l})$. Culture of the pus, blood, and pleural fluid aspirate yielded pure growth of small, smooth, non-lactose fermenting colonies on MacConkey's agar after overnight incubation, which turned pink with a metallic sheen after 48 hours and dry and wrinkled after 96 hours of incubation (Fig. 2A). Blood agar grew small, greyish white, smooth, non-haemolytic colonies after overnight incubation, 


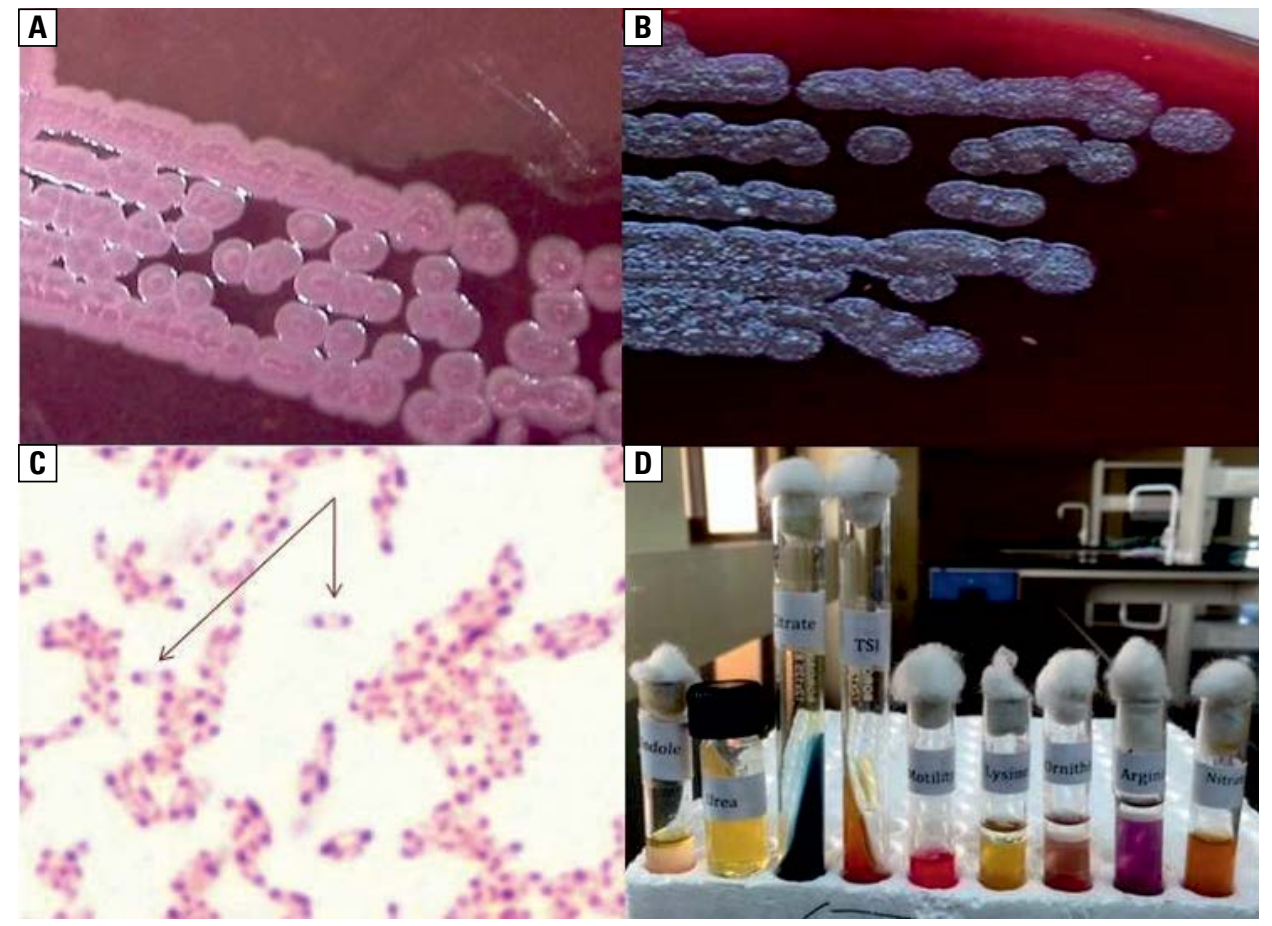

Figure 2. Identification of Burkholderia pseudomallei by (A) Growth on Mac Conkey agar, (B) Growth on Blood Agar, (C) "Safety pin" appearance on Gram stain, and (D)Biochemical reactions

which subsequently changed over to large, flat, dry, and wrinkled with central umbonation after 96 hours of incubation (Fig. 2B). Microscopy from culture revealed motile, Gram negative bacilli with typical bipolar staining giving a "safety-pin" appearance (Fig. 2C). The isolates were subsequently identified as B. pseudomallei by standard biochemical tests (positive oxidase and nitrate reduction test, positive arginine dihydrolase activity, positive citrate utilisation test, oxidative utilization of glucose, lactose, maltose, growth at $42^{\circ} \mathrm{C}$, and negative lysine decarboxylase reaction) and by their resistance to colistin $10 \mu \mathrm{g}$, gentamicin $10 \mu \mathrm{g}$, and polymyxin B 300U disks (Figs 2D, 3A). Minimum inhibitory concentration of various antibiotics by Etest (bioMérieux, Marcy l'Etoile, France) showed the isolates to be sensitive to ceftazidime, co-trimoxazole, imipenem, meropenem, and amoxicillin- clavulanate (Table 1, Fig. 3B) [5].

Once the culture report was available, ceftazidime was continued as intensive therapy ( $2 \mathrm{gm}$ IV 8-hourly) for a prolonged duration of 3 weeks followed by weight-based oral cotrimoxazole (Trimethoprim $240 \mathrm{mg}$ and Sulfamethoxazole $1200 \mathrm{mg}$ ) twice daily as maintenance therapy. Glycemic control alongwith correction of anemia and hypoalbuminemia was optimized with insulin therapy and transfusion of packed red
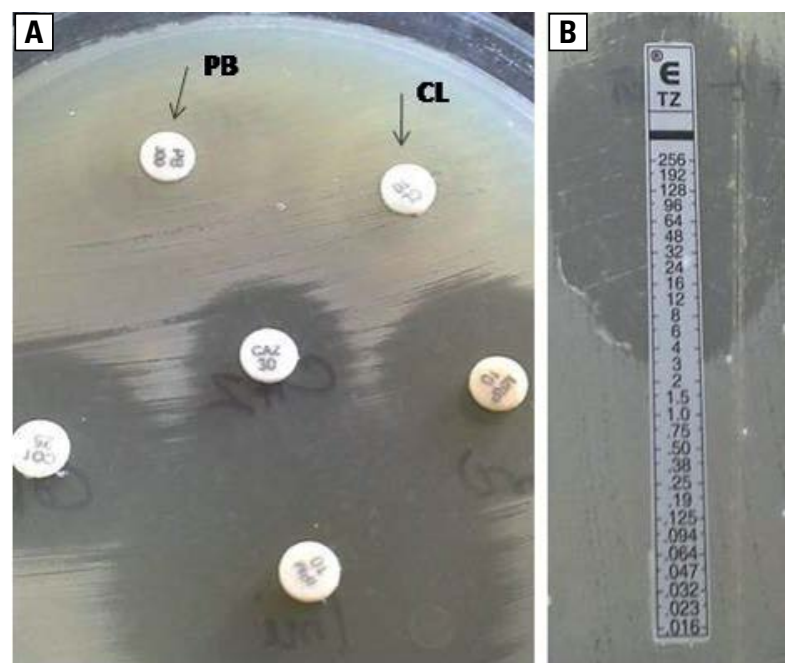

Figure 3. Antibiotic susceptibility of Burkholderia pseudomallei demonstrating (A) resistance to Polymyxin B $300 \mathrm{U}(\mathrm{PB})$ and colistin $10 \mu \mathrm{g}$ (CL) disks, and (B) Ceftazidime MIC $(3 \mu \mathrm{g} / \mathrm{ml})$ by Etest

blood cells and fresh frozen plasma respectively. Clinical improvement was noted within a week of ceftazidime therapy. A repeat blood culture after seven days of ceftazidime therapy was sterile. The patient was discharged from the hospital on $24^{\text {th }}$ day of admission with advice to continue cotrimoxazole for at least six months. On the last review visit, the patient was totally asymptomatic with complete recovery from the lesion. 
Table 1. Minimum inhibitory concentration (MIC) of antimicrobial agents for the isolates of Burkholderia pseudomallei [5]

\begin{tabular}{lcc}
\hline Antimicrobial agent & MIC $(\boldsymbol{\mu g} / \mathbf{m l})$ & $\begin{array}{c}\text { MIC interpretative criteria, for susceptible }(\boldsymbol{s}) \text {, intermediate }(\boldsymbol{i}) \text {, and resistant }(\boldsymbol{r}), \\
\text { CLSI M45-A2 }\end{array}$ \\
\hline Ceftazidime & 3 & $s \leq 8, i 16, r \geq 32$ \\
Cotrimoxazole & 2 & $s \leq 2 / 38,-, r \geq 4 / 76$ \\
Imipenem & 2 & $s \leq 4, i 8, r \geq 16$ \\
Meropenem & 2 & $s \leq 4, i 8, r \geq 16$ \\
Amoxicillin-clavulanate & 4 & $s \leq 8 / 4, i 16 / 8, r \geq 32 / 16$ \\
\hline
\end{tabular}

\section{Discussion}

Melioidosis was first recognized as a clinical entity in 1911 when Indian Bacteriologist C.S. Krishnaswami, under the guidance of the pathologist Alfred Whitmore described a "Glanders-like disease" among the morphine addicts and vagabonds found dead in the streets of Rangoon (Yangon), Burma (Myanmar) [6]. The first indigenous report of melioidosis from India was by Raghavan et al., from Mumbai in 1991 [7]. Since then, cases of human melioidosis have been reported from several Indian states [8].

Humans acquire the infection by inhalation of contaminated dust or droplets, ingestion of contaminated water, or contact with contaminated soil especially through skin abrasions $[1,2]$. People with occupational or recreational exposure to moist soil or surface water such as rice farmers, other agricultural workers, building site labourers, adventure travellers, soldiers and immigrants are at greatest risk $[1,4]$. Nearly 75 to $81 \%$ of cases occur during the rainy season [4]. The incubation period may range from days to many years; generally symptoms appear 2-4 weeks after exposure $[1,4]$. The disease can remain dormant for years and can activate many years after the initial exposure, causing diagnostic confusion [9]. Additionally, the causative agent displays inherent resistance to most of the commonly used antibiotics, penicillin, ampicillin, first-generation and second-generation cephalosporins, gentamicin, tobramycin, streptomycin, and even to polymyxin; limiting the treatment options [4].

Although healthy people may get melioidosis, the major risk factors include diabetes, heavy alcohol use, chronic pulmonary disease, chronic renal disease, thalassemia, glucocorticoid therapy, and cancer [3]. The causative bacterium is widely present in soil up to a depth of $25-45$ $\mathrm{cms}$ in the endemic regions. Their movement to the surface with the rising water table during the rainy seasons has been proposed responsible for its increased transmission rates [10]. Our patient was diabetic and developed infection in the rainy season, but was without occupational exposure to soil or water. It is possible, however, that he acquired the infection either by percutaneous inoculation by minor trivial trauma or aerosolisation of bacteria during exposure to the ongoing household construction work or use of open field for defecation.

The clinical spectrum of $B$. pseudomallei infection is extremely broad, ranging from asymptomatic infection, localized skin ulcers or abscesses, chronic pneumonia mimicking tuberculosis, and fulminant septic shock with abscesses in multiple internal organs [2-4]. Acute sepsis is the most serious, accounting for up to $60 \%$ of infections. Bacteremic dissemination is frequent and presents as multifocal pneumonia, subcutaneous abscesses, abscesses in solid abdominal visceral organs, septic arthritis, osteomyelitis, and septicaemia. On chest radiographs, diffuse nodular infiltrates often develop throughout both lungs and they coalesce, cavitate, and progress rapidly, to caseous necrosis and multiple metastatic abscess formation [3, 11, 12]. Mortality rate can reach as high as $95 \%$ in absence of effective intervention, and $50 \%$ even after antibiotic therapy [3, 12].

The remaining $40 \%$ patients have localised infections, most frequently in the lung (manifesting as mild bronchitis to severe pneumonia), and abscesses or granulomata in any organ system like cutaneous and subcutaneous abscesses, lymphadenitis, osteomyelitis, septic arthritis, liver and/or splenic abscesses, cystitis, pyelonephritis, prostatic abscesses, epididymo-orchitis, and brain abscesses [3, 8]. As evident, the clinical symptoms of melioidosis mimic those of many other diseases; thus, differentiating between melioidosis and other acute and chronic bacterial infections, including tuberculosis, is often difficult. Many patients presenting with prolonged fever, weight loss, and suspicious chest radiographic findings, 
are misdiagnosed as tuberculosis and may get anti-tubercular therapy [13] as in the present case. Around $11 \%$ of the cases present with chronic melioidosis defined as illness with symptoms for longer than 2 months' duration on presentation [3, 4]. Fifteen percent of chronic melioidosis cases present with pleural effusion [14]. The present case had acute septicaemic melioidosis, with widespread dissemination to lung (pneumonia) and subcutaneous tissue (abscess), remained undiagnosed for long, and subsequently progressed to chronic pleural involvement with pleural effusion. The CT thorax showing widespread bilateral peripheral lung nodular opacities bears evidence to disseminated 'blood-borne pneumonia' in the present case.

In tropics, melioidosis should be considered in the differential diagnosis of acute pyrexia of unknown origin, acute respiratory distress syndrome and acute onset of sepsis, especially if presenting during rainy season, if multiple pustular or necrotic skin or subcutaneous lesions develop, or if there is a radiologic pattern of tuberculosis from which tubercle bacilli cannot be demonstrated. A delay in diagnosis can be fatal, since empirical antibiotic regimens used for suspected bacterial sepsis often do not provide adequate coverage for B. pseudomallei. However, a delay in the identification of $B$. pseudomallei or a misidentification as another species (most commonly, Pseudomonas species or as a contaminant in culture) is not uncommon in laboratories that are unfamiliar with this organism [15]. It is extremely important to monitor the patients for at least one year after completion of therapy because of the risk of recurrence. Recurrent melioidosis occurs in approximately 1 in 16 patients, often in the first year after the initial presentation. Roughly a quarter of recurrences are due to reinfection, with the remainder due to relapse from a persistent focus of infection [4].

In October 2012, B. pseudomallei was upgraded to a Tier 1 Select Agent by the Centers for Disease Control and Prevention resulting in increased research and understanding of melioidosis [3]. With the expansion of endemic zone of the disease into previously considered non-endemic regions, high degree of suspicion and vigilance on the part of both microbiologists and clinicians is need of the hour for an early and accurate detection of the organism and decreasing case fatality.

\section{Conflict of interest}

The authors declare no conflict of interest.

\section{References:}

1. Cheng CA, Currie BJ. Melioidosis: epidemiology, pathophysiology, and management. Clin Microbiol Rev 2005; 18: 383-416.

2. Currie BJ, Dance DA, Cheng AC. The global distribution of Burkholderia pseudomallei and melioidosis: an update. Trans R Soc Trop Med Hyg 2008; 102 (Suppl. 1): S1-S4. doi: 10.1016/ S0035-9203(08)70002-6.

3. Currie BJ. Melioidosis: evolving concepts in epidemiology, pathogenesis and treatment. Semin Respir Crit Care Med 2015; 36:111-125. doi: 10.1055/s-0034-1398389.

4. Wiersinga WJ, Currie BJ, Peacock SJ. Melioidosis. New Engl J Med 2012; 367: 1035-1044. doi: 10.1056/NEJMra1204699.

5. Clinical and Laboratory Standards Institute. 2010. Methods for Antimicrobial Dilution and Disk Susceptibility Testing of Infrequently Isolated or Fastidious Bacteria; approved guideline, $2^{\text {nd }}$ edition. M45-A2, vol. 30, no 18, Clinical and Laboratory Standards Institute, Wayne, PA, USA.

6. Whitmore A. An account of a glanders-like disease occurring in Rangoon. J Hyg 1913; 13: 1-34.

7. Raghavan KR, Shenoi RP, Zaer F, Aiyer R, Ramamoorthy P, Mehta MN. Melioidosis in India. Indian Pediatr 1991; 28: 184-188.

8. Vidyalakshmi K, Lipika S, Vishal S, Damodar S, Chakrapani M. Emerging clinic-epidemiological trends in melioidosis: analysis of 95 cases from western coastal India. Int J Infect Dis 2012; 16: e491-497. doi: 10.1016/j.ijid.2012.02.012.

9. Badsha H, Edwards CJ, Chng HH. Melioidosis in systemic lupus erythematosus: the importance of early diagnosis and treatment in patients from endemic areas. Lupus 2001; 10: 821-823.

10. Sood S, Khedar RS, Joad SH, Gupta R. Septicaemic melioidosis: case report from a non-endemic area. J Clin Diagn Res 2014; 8: DD01-2. doi: 10.7860/JCDR/2014/9684.5257.

11. Saravu K, Mukhopadhyay C, Vishwanath S et al. Melioidosis in Southern India: Epidemiological and clinical profile. Southeast Asian J Trop Med Public Health 2010; 41: 401-409.

12. White NJ. Melioidosis. Lancet 2003; 361: 1715-1722.

13. Barman P, Sidhwa H, Shirkhande PA. Melioidosis: a case report. J Glob Infect Dis 2011; 3: 183-186. doi: 10.4103/0974777X.81697.

14. Neliyathodi S, Thazhatethil AN, Pallivalappil L, Balakrishnan D. Pleuropulmonary melioidosis with osteomyelitis rib. Lung India 2015; 32: 68-69. doi: 10.4103/0970-2113.148456.

15. Peacock SJ, Schweizer HP, Dance DA, Smith TL, Gee JE, Wuthiekunan V, et al. Management of accidental laboratory exposure to Burkholderia pseudomallei and B. mallei. Emerg Infect Dis 2008; 14 (7):e2. doi: 10.3201/eid1407.071501. 